\title{
A New Computer-Based Tool to Reduce Potentially Inappropriate Prescriptions in Hospitalized Geriatric Patients
}

\author{
Anna Maria Grion ${ }^{1}$ Umberto Gallo ${ }^{1} \cdot$ Daniel Dumitru Tinjala $^{1} \cdot$ Julia Daragjati $^{2}$. \\ Michele Loreggian $^{2}$ - Giovanna Cardaci ${ }^{2}$ Arduino Mangoni ${ }^{3}$ - Alberto Pilotto ${ }^{2,4}$
}

Published online: 20 January 2016

(c) Springer International Publishing Switzerland 2016

\begin{abstract}
Background Potentially inappropriate prescriptions (PIPs), associated with adverse drug reactions, hospitalization, and wasteful healthcare spending, are common in elderly patients with comorbidities and multiple drugs.

Objective Our objective was to develop and validate a new tool to reduce PIPs in a hospitalized geriatric population.

Methods This was an observational cohort study of two cohorts (development $[n=100$ subjects] and validation $[n=449$ subjects $]$ ) of consecutive patients aged $\geq 65$ years admitted to geriatric wards from April to December 2012 . In the development phase, data on clinical and demographic characteristics, Multidimensional Prognostic Index (MPI), and prescribed drugs before hospital admission were collected and processed using a tool that integrates the Screening Tool of Older Persons' Potentially Inappropriate Prescriptions (STOPP) criteria and the Micromedex ${ }^{\mathrm{TM}}$ Drug-Reax System, a drug-management platform. This tool generated a report that was provided to the treating physicians. The weight of the PIPs, as defined by the Medication Appropriateness Index (MAI), was
\end{abstract}

Alberto Pilotto

alberto.pilotto@galliera.it

1 Pharmaceutical Department, Local Health Unit n. 16, Padua, Italy

2 Geriatrics Unit, S. Antonio Hospital, Local Health Unit n. 16, Padua, Italy

3 Department of Clinical Pharmacology, School of Medicine, Flinders University, Adelaide, SA, Australia

4 Department of Geriatric Care, OrthoGeriatrics and Rehabilitation-Frailty Area, E.O. Galliera Hospital, National Relevance and High Specialization Hospital, Mura delle Cappuccine 14, 16128 Genoa, Italy assessed on admission and on discharge. Similar procedures were followed in the validation cohort.

Results PIPs were independently associated with polypharmacy and with prescribing of antithrombotics, sedatives and antidepressants in both cohorts. The use of the tool led to a significant reduction of the MAI score, both in the development (median score 4 [interquartile range; IQR $1-5$ ] vs. 2 [IQR $0-4$ ], $p<0.001$ ) and in the validation cohorts (median 3 [IQR 1-5] vs. 2 [IQR 0-4], $p<0.001$ ).

Conclusion This tool significantly reduced PIPs, as defined by the MAI score, in a hospitalized geriatric population. This strategy might be useful to minimize inappropriate medication exposure in this group.

\section{Key Points}

A new computer-based tool has been developed and validated to detect potentially inappropriate prescriptions (PIPS) in elderly hospitalized patients.

The tool utilizes a multidisciplinary approach involving physicians and the hospital pharmacist.

Use of the tool resulted in a reduction in PIPs at discharge in this pilot study.

\section{Introduction}

Older patients are more at risk of medication-related adverse events, e.g., adverse drug reactions (ADRs), in view of frequent comorbidity, complex drug regimens, and age-related changes in pharmacokinetics and pharmacodynamics. Potentially inappropriate prescriptions (PIPs), 
defined as medications whose risks outweigh benefits in a patient, are known to predispose to medication-related adverse events [1]. PIPs also refers to the use of unnecessary drugs (absence of indication or duplication) or of drugs prescribed at an inappropriate dosage or for an inappropriate length of time. PIPs are highly prevalent among elderly individuals, particularly those hospitalized for acute illnesses [2, 3]. Some classes of drugs are considered inappropriate, or potentially inappropriate, in geriatric patients because of the relatively high risk of pharmacokinetic and/or pharmacodynamic drug-drug interactions (DDIs) or drug-disease interactions (DDSIs).

Available computerized PIPs software programs have different processing and reporting criteria. This reflects, at least in part, the discordance observed with regard to drug interaction reports [4]. Moreover, clinically important DDSIs can be evaluated using different explicit or implicit criteria. In view of the high risk of medication-related adverse events, hospitalization, and wasteful healthcare, specific criteria have been developed to identify medications that are considered inappropriate in this population. The most popular criteria include the Beers' list [5] and the Screening Tool of Older Persons' Potentially Inappropriate Prescriptions (STOPP) criteria [6].

Although the exact clinical role of these criteria remains unclear [7], early detection of PIPs can prevent or limit medication-related adverse events and improve geriatric care $[8,9]$. Therefore, interventions to optimize prescribing in this population are considered a public health priority. Several computer-based systems (Computerized Provider Order Entry [CPOE]) have been developed to improve prescription appropriateness and clinical outcomes [10, 11]. However, none of the available systems incorporate validated and integrated criteria to detect PIPs, DDIs, and/ or other forms of inappropriate prescribing.

We sought to address this issue by developing and validating a new tool to reduce PIPs in older patients admitted to a geriatric unit.

\section{Subjects and Methods}

\subsection{Subjects}

This was a pilot study tested in a geriatric ward. In this prospective study, all patients aged $\geq 65$ years consecutively admitted to the Geriatric Unit of S. Antonio Hospital (Padua, Italy) due to acute illness or relapse of chronic illness were screened for inclusion in the study. All screened patients were informed of the nature of the study. Exclusion criteria were as follows: patients who were terminally ill or had advanced cancer or refused to participate in the study. Patients with missing data at discharge due to in-hospital death or transfer to other wards were also excluded from the analyses. Two cohorts of patients were included in the study. The first cohort of 100 patients was recruited from 1 April to 15 May 2012 for tool development; the second cohort of 449 patients was recruited from 16 May to 31 December 2012 for tool validation. All physicians of the geriatric wards participated in the study. At baseline, the physician completed a survey form that included the following data: (1) demographic details (date of birth, sex, individual code); (2) current and past medical conditions; (3) frequency of falls in the previous month; (4) functional, nutritional, and bio-psycho-social assessment according to the Multidimensional Prognostic Index (MPI); (5) medications taken at home prior to hospitalization. Drug information included the Anatomical Therapeutic Chemical (ATC) classification, formulation, dose, frequency, and treatment duration. Short-term therapies (e.g., antibiotics) and topical medications (e.g., eye drops) were not considered. Table 1 shows demographic, clinical, and drug use data for patients included in the two cohorts.

\subsection{Study Design}

At baseline, all data regarding demographics, drugs used, and comorbidities were entered by a pharmacist into the computer-based tool, which integrates the STOPP criteria and the Micromedex ${ }^{\mathrm{TM}}$ Drug-Reax System, a validated drug-management platform. On the basis of these data, the tool generated a report that included all PIPs as derived from the computer-based analyses. Whenever a PIP was detected, the pharmacist provided the report to the physician in charge of the patient. The physician was asked to address the questions relating to therapy selection in discussion during the daily briefing. Thus, therapeutic decisions were taken by physicians on the basis of clinical conditions of patients and the computer-generated report as discussed with the pharmacist. When no PIPs were detected by the tool, no report was generated and consequently no interaction occurred between the pharmacist and the physicians who wrote prescriptions without the support of the computer-based tool. To evaluate the change in PIPs between admission and discharge from the hospital, the number of PIPs, as defined by the Medication Appropriateness Index (MAI), were assessed on admission and on discharge. This study design was adopted for both the development and the validation cohorts (Fig. 1).

\subsection{System Development}

This tool was developed in Microsoft ${ }^{\circledR}$ Access, 2003 version (Microsoft Corporation, Redmond, WA, USA), by staff at our institution. Demographic data, medical history, and drugs taken chronically before hospital admission were 
Table 1 Characteristics of patients in the development and validation cohorts

\begin{tabular}{lccc}
\hline Characteristic & $\begin{array}{l}\text { Development cohort } \\
(n=100)\end{array}$ & $\begin{array}{l}\text { Validation cohort } \\
(n=449)\end{array}$ & $p$ value \\
\hline Males & $48(48.0)$ & $178(39.6)$ & 0.125 \\
Females & $52(52.0)$ & $271(60.4)$ & \\
Age, years & $87(79.75-90.25)$ & $86(81-90)$ & 0.843 \\
Diagnosis & & & \\
Hypertension & $68(68.0)$ & $324(72.2)$ & 0.405 \\
Arrhythmia & $37(37.0)$ & $161(35.9)$ & 0.829 \\
Ischemic cardiomyopathy & $35(35.0)$ & $129(28.7)$ & 0.215 \\
Dementia & $32(32.0)$ & $189(42.1)$ & 0.063 \\
COPD & $22(22.0)$ & $88(19.6)$ & 0.587 \\
Diabetes & $21(21.0)$ & $106(23.6)$ & 0.576 \\
Stroke or TIA & $14(14.0)$ & $82(18.3)$ & 0.310 \\
Chronic kidney disease & $14(14.0)$ & $82(18.3)$ & 0.090 \\
Depression & $12(12.0)$ & $56(11.1)$ & 0.805 \\
MPI score & $0.72(0.56-0.81)$ & $0.69(0.50-0.81)$ & 0.059 \\
Number of drugs and PIPs & & & \\
Number of drugs at admission & $6(4-8)$ & $6(4-8)$ & 0.969 \\
PIPs at admission/patient & $1(0-2)$ & $244(54)$ & 0.577 \\
STOPP criteria & $53(53)$ & $167(37)$ & 0.825 \\
Major DDI & $38(38)$ & $67(67)$ & \\
PIPs & & & 0.909 \\
Data & $(70)$ & 0.551
\end{tabular}

Data are presented as $n(\%)$ or median (IQR)

$C O P D$ chronic obstructive pulmonary disease, $D D I$ drug-disease interaction, $I Q R$ interquartile range, $M P I$ multidimensional prognostic index, PIPs potentially inappropriate prescriptions, STOPP Screening Tool of Older Person's Prescriptions, TIA transient ischemic attack entered into the software. A report was generated on all PIPs identified by the STOPP criteria and/or clinically relevant DDIs as identified by the Micromedex ${ }^{\mathrm{TM}}$ DrugReax platform. Relevant DDIs were defined as major or contraindicated DDIs, while minor and moderate DDIs were excluded from the system. STOPP, a medicine review tool, is a validated instrument organized according to organ systems and including 65 criteria for potentially inappropriate prescribing. These criteria, validated using the Delphi consensus technique by 18 experts in geriatric pharmacotherapy from the UK and Ireland [6], identify PIPs related to DDSIs, DDIs, drug class duplication, dose, and therapeutic time window. The STOPP criteria are able to identify a significant proportion of patients requiring hospitalization because of PIP-related adverse events [16]. As only three of the 65 STOPP criteria detect clinically relevant DDIs, we also used the Micromedex ${ }^{\mathrm{TM}}$ Drug-Reax program, version 2.0 [17], a drug interaction compendia commonly used by drug information services to identify DDIs [18]. Moreover, Micromedex provides information about the risk of PIPs or ADRs due to co-administration of drugs and the interaction mechanisms. All potential clinically important DDIs identified by Micromedex are classified according to their degree of severity as 'contraindicated' or 'major'. DDI potential risk was defined according to the Medical Dictionary for Regulatory Activities, version 9.1 (MedDRA) [19]. When a pair of drugs was not listed in the Micromedex database, the interaction was not considered further.

\subsection{Assessment of Potentially Inappropriate Prescriptions (PIPs)}

This tool was used to identify patients' PIPs on admission to and on discharge from the hospital. A report was generated for each PIP identified in a patient. The pharmacist and the treating physician discussed the report and made a decision regarding continuing or stopping specific drugs (Fig. 2).

Prescription appropriateness was assessed using the MAI, which was specifically developed in older adults and is considered a reliable tool in a number of clinical settings [20]. The original MAI used ten weighted criteria in the form of questions for individual drugs; it produces a score for each drug, and a total score for a patient, using the following definitions: 'appropriate', 'marginally appropriate, or 'inappropriate' [20]. Medications were dichotomized in this study as being either 'appropriate' or 'inappropriate' (if considered a PIP by the tool). Similar to the original MAI, the following scores were assigned for 


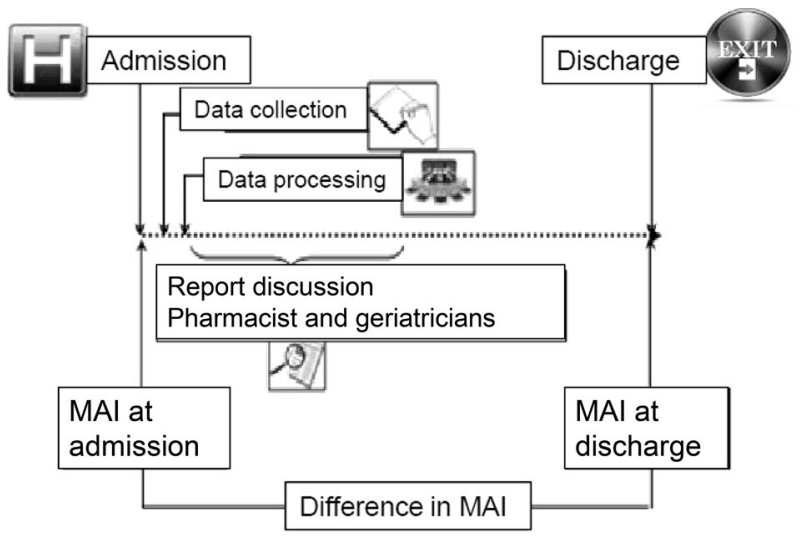

Fig. 1 Study design. MAI Medication Appropriateness Index

each PIP: absence of indication $(+3)$, inappropriate dose $(+2)$, DDI $(+2)$, DDSI $(+2)$, inappropriate length of treatment $(+1)$, and duplicate prescription $(+1)$.

The difference in the number of PIPs before and after intervention was evaluated by calculating the difference between the MAI score on admission and on discharge.

\subsection{Multidimensional Prognostic Index (MPI)}

Patients' frailty was assessed using the MPI, a validated prognostic tool based on a standard Comprehensive Geriatric Assessment (CGA) that predicts short (1 month) and long-term (1 year) mortality in elderly subjects [12]. The MPI has shown good calibration and discriminatory ability to evaluate mortality risk in hospitalized geriatric patients [13-15].

The MPI was calculated using CGA data by summing the total scores of eight domains each, given a value between 0.0 (lowest risk) and 1.0 (maximum risk of mortality). The eight domains included basal Activities of Daily Living (ADL); Instrumental ADL (IADL); cognitive status using the Short Portable Mental Status Questionnaire (SPMSQ); nutritional status evaluated using the Mini Nutritional Assessment (MNA); risk of developing pressure sores assessed by the Exton Smith Scale (ESS); multimorbidity evaluated with the Cumulative Index Rating Scale (CIRS); number of drugs being taken; and co-habitation status (alone, with family, or in an institution). According to previously validated cut-off values, three MPI categories were identified, i.e., $0-0.33$ (low risk), 0.34-0.66 (moderate risk), and 0.67-1.0 (severe risk of mortality) [12].

\subsection{Statistical Analysis}

Statistical analysis was performed using R-project software version 2.15.1 for Windows (GNU project) [21].
Descriptive statistics included mean and standard deviation for normally distributed variables and median and interquartile range (IQR) for nonparametric variables. Study groups were compared using chi-squared or Fisher's exact test for categorical variables, the Student's $t$ test for normally distributed continuous variables, and the Wilcoxon rank-sum test for nonparametric variables. The Mann-Whitney and Kruskal-Wallis tests were used to determine the independence of two or more nonparametric variables, respectively.

We performed a multivariate analysis to determine patient-related characteristics associated with inappropriate medication use. Because PIPs were commonly reported in our study population ( $>10 \%$ ), adjusted odds ratios (ORs) could not be used to approximate the relative risks (RRs). Instead, the Zhang and Yu method was applied to estimate the RRs [22]. $P$ values of $<0.05$ were considered statistically significant for all tests.

\section{Results}

\subsection{Study Population}

For the first cohort, a population of 137 patients was screened for inclusion. During the enrolment period, ten patients died during hospitalization, 16 patients were transferred to other wards, and 11 patients who were terminally ill or had advanced cancer were excluded from the analyses. For the second cohort, an initial 592 patients were screened for inclusion. During the enrolment period, 54 patients died during hospitalization, 28 were transferred to other wards, and 61 patients who were terminally ill or had advanced cancer were excluded from the analyses.

Thus, the first cohort included 100 patients recruited from 1 April to 15 May 2012, and the second cohort included 449 patients recruited from 16 May to 31 December 2012.

\subsection{System Development}

Table 1 outlines the characteristics of patients included in the development cohort according to sex, age, medical condition, MPI score, number of drugs, and PIPs identified on admission. The tool identified PIPs in $67 \%$ of patients on admission. Specifically, $53 \%$ of patients fulfilled at least one STOPP criterion and $38 \%$ had at least one DDI. The median number of PIPs detected by the tool was 1 (IQR 0-2). On admission, factors independently associated with PIPs (Table 2) were poly-pharmacy (defined as five or more drugs; OR 2.48; $95 \%$ confidence interval [CI] 1.44-4.27) and use of antithrombotic agents (OR 2.06; $95 \%$ CI 1.17-3.61), psycholeptics and/or antidepressants 
SAFE Project Report

Source: Micromedex and STOPP criteria 2008

\begin{tabular}{|c|c|c|c|c|c|c|c|}
\hline Physician: & \multicolumn{2}{|c|}{ Aaaa*Bbbb } & & \multicolumn{2}{|c|}{ Bed number: 3} & & \multirow{4}{*}{$\begin{array}{l}\text { Clinical Management } \\
\text { When paroxetine and an antiplatelet agent } \\
\text { are given concurrently, monitor patient for } \\
\text { signs of increased bleeding }\end{array}$} \\
\hline $\begin{array}{l}\text { Patient: } \\
\text { Tax code: }\end{array}$ & \multicolumn{3}{|c|}{$\begin{array}{l}\text { Xxxx*Yyyy } \\
\text { XXXYYY33A41G279P }\end{array}$} & & & & \\
\hline Drug 1 & Drug 2 & \multirow{2}{*}{\multicolumn{2}{|c|}{$\begin{array}{l}\text { Warning } \\
\text { Concurrent use of } \\
\text { PAROXETINE and } \\
\text { ANTIPLATELET AGENTS may } \\
\text { result in an increased risk of } \\
\text { bleeding. }\end{array}$}} & Severity & Documentation & Onset & \\
\hline Aspirin & Paroxetine & & & Major & Good & Non specified & \\
\hline Alprazolam & Digoxin & \multicolumn{2}{|c|}{$\begin{array}{l}\text { Concurrent use of } \\
\text { ALPRAZOLAM and DIGOXIN } \\
\text { may result in digoxin toxicity } \\
\text { (nausea, vomiting, } \\
\text { arrhythmias). }\end{array}$} & Major & Good & Delayed & $\begin{array}{l}\text { Monitor for signs of digoxin intoxication (eg, } \\
\text { nausea, vomiting, diarrhea, persistent } \\
\text { headache, confusion, fainting, visual } \\
\text { disturbances). If symptoms are present, } \\
\text { obtain a digoxin level and reduce dose } \\
\text { accordingly. }\end{array}$ \\
\hline Aspirin & Warfarin & \multicolumn{2}{|c|}{$\begin{array}{l}\text { Concurrent use of WARFARIN } \\
\text { and ANTIPLATELET AGENTS } \\
\text { may result in increased risk of } \\
\text { bleeding. }\end{array}$} & Major & Excellent & Delayed & $\begin{array}{l}\text { Coadministration of warfarin and antiplatelet } \\
\text { agents may increase the risk of bleeding. } \\
\text { Monitor patients closely for signs or } \\
\text { symptoms of bleeding and evaluate } \\
\text { promptly. Lab monitoring may be } \\
\text { appropriate. }\end{array}$ \\
\hline \multicolumn{2}{|c|}{ Medicinal } & & \multicolumn{2}{|l|}{ Active Agent } & \multicolumn{2}{|c|}{ STOPP criteria } & Comment \\
\hline \multicolumn{3}{|c|}{ CARDIOASPIRIN 30 tablet film-coated } & ACETYLSALICY & IC ACID & \multicolumn{2}{|c|}{$\begin{array}{l}\text { Aspirin with no history of coronary, } \\
\text { cerebral or peripheral vascular } \\
\text { symptoms or occlusive event }\end{array}$} & Non indicated \\
\hline \multicolumn{3}{|c|}{ LANOXIN 30 tablet $0.250 \mathrm{mg}$} & DIGOXIN & & \multicolumn{2}{|c|}{$\begin{array}{l}\text { Digoxin }>125 \mu \mathrm{g} \text { per day with } \\
\text { impaired renal function }\end{array}$} & Digoxin toxicity \\
\hline \multicolumn{3}{|c|}{ BRUFEN 30 tablet $400 \mathrm{mg}$ film-coated } & IBUPROFEN & & \multicolumn{2}{|c|}{ NSAID with chronic renal failure } & Acute renal failure \\
\hline
\end{tabular}

Fig. 2 Example of report on the basis of data from STOPP criteria and the Micromedex system as provided by the computer-based tool used in the present study. NSAIDs nonsteroidal anti-inflammatory

(OR 2.00; $95 \%$ CI 1.03-3.86). As shown in Fig. 3, using this tool led to a significant reduction in the median MAI score from 4 on admission (IQR 1-5) to 2 on discharge (IQR 0-4) $(p<0.001)$.

\subsection{System Validation}

As outlined in Table 1, no significant differences were observed between the development and validation cohorts according to sex distribution, age, clinical conditions, MPI score, number of medications, and PIPs identified on admission. At least one PIP was identified in 314 of the 449 patients $(70 \%)$ in the validation cohort. In particular, PIPs were identified in 244 patients (54\%) on the basis of STOPP criteria, while relevant DDIs were identified in 167 patients (37\%). Polypharmacy (OR 3.01; $95 \%$ CI 2.30-3.94) and use of antithrombotic agents (OR 2.34; $95 \%$ CI 1.77-3.09), psycholeptics and/or antidepressant drugs (OR 1.84; $95 \%$ CI 1.37-2.47) were also significantly associated with an increased risk of PIPs in this cohort (Table 2). drugs, STOPP Screening Tool of Older Persons' Potentially Inappropriate Prescriptions

As outlined in Fig. 3, there was a significant decrease in the median MAI score from 3 (IQR 1-5) on admission to 2 (IQR $0-4 ; p<0.001$ ) on discharge.

\subsection{Differences in PIPs}

No significant differences in the median MAI score on admission were detected between the two cohorts (development 4, IQR 1-5 vs. validation 3, IQR 1-5, $p>0.05$ ). The MAI score improved (decreased) after the intervention in $61.9 \%$ of patients with PIPs at admission. By contrast, the MAI score worsened (increased) in $14.7 \%$ of patients. As shown in Table 3, patients without PIPs on admission were exposed to a twofold higher risk of a worse MAI score at discharge than patients admitted with PIPs (26.2 vs. $14.7 \%$; OR 2.1 ; $95 \%$ CI $1.3-3.2$ ).

We observed an improvement in 175 of 297 patients (58.9\%) according to STOPP criteria and in 131 of 205 patients $(63.9 \%)$ according to the DDIs identified by Micromedex. 
Table 2 Multivariate analysis of variables independently associated with potentially inappropriate prescriptions at admission: demographic characteristics, grade of polytherapy and Multidimensional Prognostic Index, diagnosis, and drugs

\begin{tabular}{|c|c|c|}
\hline Variable & $\begin{array}{l}\text { Development cohort } \\
(n=100)\end{array}$ & $\begin{array}{l}\text { Validation cohort } \\
(n=449)\end{array}$ \\
\hline Sex (males vs. females) & $1.02(0.58-1.79)$ & $0.81(0.60-1.09)$ \\
\hline Age $(65-75$ vs. $\geq 80$ years of age $)$ & $1.71(0.96-3.05)$ & $0.94(0.66-1.34)$ \\
\hline Polytherapy (0-4 vs. $\geq 5$ drugs) & $2.48(1.44-4.27)$ & $3.01(2.30-3.94)$ \\
\hline Grades MPI (MPI 1 vs. MPI 2-3) & $1.11(0.56-2.20)$ & $1.43(1.00-2.04)$ \\
\hline \multicolumn{3}{|l|}{ Diagnosis } \\
\hline COPD & $0.75(0.40-1.41)$ & $1.49(0.99-2.23)$ \\
\hline Ischemic cardiomyopathy & $1.70(0.91-3.18)$ & $0.89(0.66-1.21)$ \\
\hline Stroke or TIA & $0.73(0.35-1.51)$ & $0.98(0.70-1.40)$ \\
\hline Diabetes & $0.99(0.50-1.96)$ & $1.08(0.77-1.52)$ \\
\hline Parkinson's disease & $0.46(0.20-1.07)$ & $1.16(0.73-1.84)$ \\
\hline Chronic kidney disease & $1.63(0.62-4.28)$ & $1.28(0.86-1.80)$ \\
\hline Hypertension & $1.21(0.62-2.17)$ & $1.34(1.00-1.80)$ \\
\hline Dementia & $1.08(0.59-2.93)$ & $1.03(0.70-1.52)$ \\
\hline \multicolumn{3}{|l|}{ Drugs (ATC code) } \\
\hline Drugs used in diabetes (A10) & $0.76(0.38-1.51)$ & $0.94(0.67-1.34)$ \\
\hline Antithrombotic agents (B01) & $2.06(1.17-3.61)$ & $2.34(1.77-3.09)$ \\
\hline Cardiac glycosides (C01A) & $1.49(0.57-3.94)$ & $1.38(0.83-2.31)$ \\
\hline $\begin{array}{l}\text { Antihypertensive agents }(\mathrm{C} 02, \mathrm{C} 03 \mathrm{~A}, \mathrm{C} 03 \mathrm{~B} \text {, } \\
\mathrm{C} 03 \mathrm{C}, \mathrm{C} 07, \mathrm{C} 08 \mathrm{C}, \mathrm{C} 09)\end{array}$ & $1.31(0.66-2.61)$ & $1.34(1.00-1.80)$ \\
\hline Lipid-modifying agents (C10) & $1.27(0.61-2.62)$ & $1.35(0.87-2.12)$ \\
\hline NSAID (M01A) & $0.99(0.19-5.09)$ & $2.07(0.81-5.27)$ \\
\hline Opioids (N02A) & $0.81(0.34-1.90)$ & $1.87(0.96-3.68)$ \\
\hline Psycholeptics (N05)/antidepressants (N06A) & $2.00(1.03-3.86)$ & $1.84(1.37-2.47)$ \\
\hline
\end{tabular}

Data are presented as relative risk (95\% confidence interval)

$A T C$ anatomical therapeutic chemical classification system, COPD chronic obstructive pulmonary disease, $M P I$ multidimensional prognostic index, NSAIDs nonsteroidal anti-inflammatory drugs, PIP potentially inappropriate prescribing, $R R$ relative risk, $T I A$ transient ischemic attack
Fig. 3 Medication Appropriateness Index at admission and at discharge. White circles indicate outliers. $I Q R$ interquartile range, $M A I$ Medication Appropriateness Index

\section{Discussion}

Elderly individuals often have numerous coexisting diseases requiring pharmacological treatment. The effects of aging on drug metabolism are nevertheless complex. As a result, prescribing therapy for older patients presents

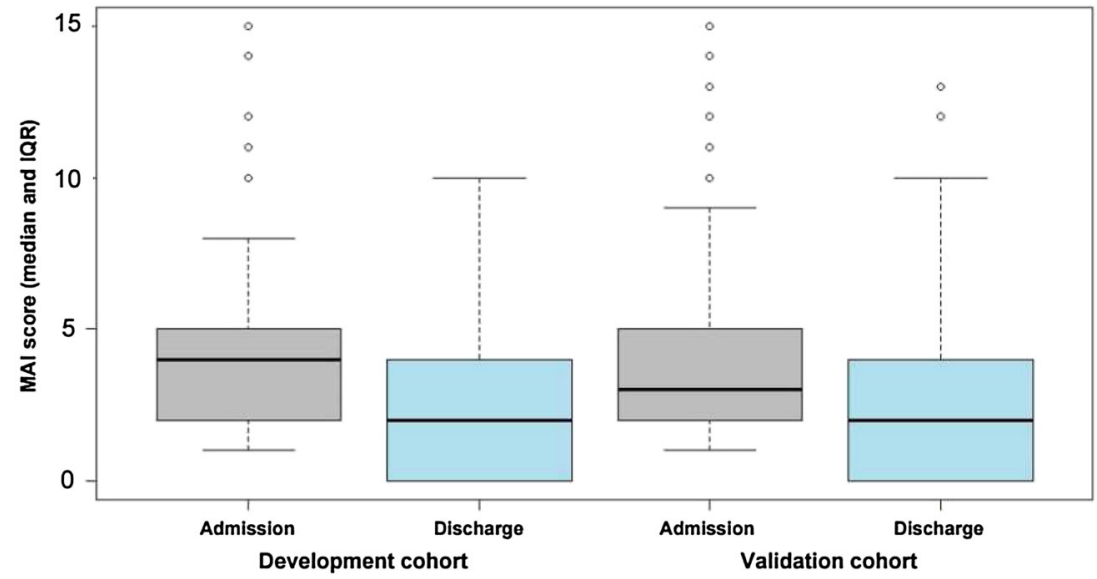

unique challenges. In addition, a large number of new drugs are introduced yearly, causing an ever-increasing risk of drug interactions.

Several consensus-based guidelines and criteria for appropriate prescribing in elderly patients have been developed to reduce inappropriate prescriptions; for example, the 
Table 3 Change in medication appropriateness index score

\begin{tabular}{|c|c|c|c|c|}
\hline & \multicolumn{3}{|c|}{ Total $(n=549)$} & \\
\hline & $\begin{array}{l}\text { Patients with } \\
\text { improvement }\end{array}$ & $\begin{array}{l}\text { Patients with } \\
\text { no change }\end{array}$ & $\begin{array}{l}\text { Patients with } \\
\text { deterioration }\end{array}$ & \\
\hline Patients with PIPs at admission $(n=381)$ & $236(61.9 \%)$ & $89(23.4 \%)$ & $56(14.7 \%)$ & \\
\hline Patients without PIPs at admission $(n=168)$ & - & $124(73.8 \%)$ & $44(26.2 \%)$ & $\begin{array}{l}26.2 \text { vs. } 14.7 \% \\
\text { OR } 2.1 \\
95 \% \text { CI } 1.3-3.2\end{array}$ \\
\hline
\end{tabular}

$C I$ confidence interval, MAI medication appropriateness index, $O R$ odds ratio, PIP potentially inappropriate prescribing

Beers' criteria were developed to assist healthcare professionals to improve the safety of prescribing medications in this group. The STOPP criteria were recently developed and specifically validated to decrease the number of PIPs in older hospitalized patients [23]. Recent studies comparing the STOPP and Beers' criteria reported that the former were more sensitive than the latter [16]. In one study, STOPP identified a significantly higher proportion of patients requiring hospitalization consequent to PIP-related adverse events than did the Beers' criteria [3]. In fact, recent reports have described the efficacy of STOPP criteria in identifying potentially avoidable ADRs or drug-related hospital readmissions [9, 24].

As only three of the 65 criteria relate to DDIs, the STOPP tool does not provide a comprehensive list of clinical DDIs known to be associated with an increased risk of ADRs (e.g., hyperkalemia due to drug interactions or DDIs that increase digoxin toxicity) [25].

Nevertheless, DDIs are potentially life-threatening events in older people receiving medications. Reporting on geriatric outpatients taking more than one drug and attending a day clinic, Tulner et al. [26] noted that $25 \%$ of these patients had adverse events or diminished treatment effectiveness that may have been at least partially caused by those DDIs. In a recent review of 17 population-based studies on the prevalence and outcomes related to potentially harmful DDIs in older people, exposure to DDIs was associated with increased risk of hospitalization [27].

While the most frequently used tools for detecting PIPs-namely Beers criteria [1, 5, 28], STOPP criteria [3, 6, 23], Improved Prescribing in the Elderly Tool (IPET) [29], Silberstein Lipton criteria [30], Assessing Care of Vulnerable Elders (ACOVE) [31], MAI [20] and prescription-only medicines (POM) [32]—are specifically oriented to detecting DDIs or DDSIs, very few of them detect both DDIs and DDSIs. Although each one has its own strengths and weaknesses, very few of the available DDI screening programs can be considered ideal [5, 20, 28-33]. We chose the Micromedex ${ }^{\mathrm{TM}}$ Drug-Reax system to detect potential DDIs that can cause clinically relevant interactions because of its reported good sensitivity and specificity [34]. Sensitivity was defined as the software's ability to correctly identify clinically important interaction pairs, and specificity was defined as its ability to ignore interaction pairs that were not clinically relevant. Moreover, Micromedex ${ }^{\mathrm{TM}}$ Drug-Reax is one of the few software platforms providing structured information on the frequency of, severity of, and clinical management strategies to manage the side effects of DDIs [35].

Our tool was developed based on the STOPP criteria, specifically developed and validated for an older population, and from the Micromedex ${ }^{\mathrm{TM}}$ Drug-Reax System, which identifies clinically relevant DDIs. To our knowledge, this is the only electronic tool that integrates information on PIPs from validated explicit criteria and a druginteraction detection database.

The tool was developed and validated using a cohort of consecutively hospitalized patients. This was an ideal setting to test strategies to reduce inappropriate medication, because patients are evaluated daily and medical records provide a vast amount of updated information concerning socio-demographic and clinical characteristics. The selected patients can be considered representative of a frail, hospitalized geriatric population with a relatively high prevalence of comorbidity, disability, and multiple drug regimens, increasing the risk of $\mathrm{ADR}$ and other medication-related adverse events [36].

We found the development and validation cohorts to have similar demographic and clinical characteristics, including the number of drugs and PIPs identified on admission. PIPs were independently associated with polypharmacy, antithrombotic, psycholeptic and/or antidepressant drugs in both cohorts. Several studies have reported that prescription of five or more drugs is associated with a significant risk of inappropriate medication use, adverse drug events [24], and/ or suboptimal healthcare spending [37]. In agreement with other studies, we found that PIPs were associated with antithrombotic drug use (antiplatelet agents, warfarin or heparin). For example, Budnitz et al. reported that antithrombotic drugs are implicated in nearly half of the 100,000 emergency hospitalizations for adverse drug events in US older adults each year [38]. In addition, our data concur 
with recent studies reporting that the use of antidepressants or neuroleptics are associated with increased risk of several adverse outcomes such as falls and stroke in older people [39, 40].

Study findings also highlight the important role of a pharmacist-led tool in improving prescription appropriateness. In fact, the intervention reduced the use of (1) unnecessary drugs (absence of indication or duplicate prescription), (2) the risk of DDIs and DDSIs, and (3) the prescription of drugs at inappropriate doses or duration in the selected patients.

Although several studies have evaluated PIPs computerized software programs in older adults, methodological weaknesses, including population and intervention heterogeneity, have not allowed comprehensive meta-analysis approaches to determine the clinical value of individual platforms [41, 42].

\subsection{Study Strengths}

Our study had several strengths

1. Development and validation of a new computerized tool integrating criteria from established instruments (Micromedex $^{\mathrm{TM}}$ Drug-Reax System and STOPP) addressing different aspects of appropriate prescribing. To our knowledge, very few previous studies included different criteria in a single integrated evaluation.

2. Inclusion of a validated MPI to identify patients with PIPs at high risk for iatrogenic diseases.

3. The indirect demonstration that the application of this tool and the intervention of the pharmacist could play a key role in reporting prescription errors as well as assisting in decisions regarding pharmacological management of these complex patients. Since the report was created and discussed only when the tool detected PIPs, the finding that patients without PIPs on admission were exposed to a twofold higher risk of a worse MAI score at discharge than patients admitted with PIPs indirectly suggests a positive effect of this system in improving MAI.

To increase both safety and efficacy by reducing the probability of ADRs, incorrect drugs were changed or discontinued. The pharmacist did not just enter data and process the reports. Rather, this healthcare professional also actively contributed to therapy prescriptions, supporting the physicians with his/her pharmacological knowledge and vigilance [7, 9].

\subsection{Study Limitations}

Our study also includes some limitations. As it was carried out in a single center, further studies need to be conducted in other healthcare settings (e.g., primary care and nursing homes) to confirm the clinical utility of this tool. Second, the analysis did not include over-the-counter (OTC) medications and drugs used to treat acute illness (e.g., antibiotics). It is not possible to rule out that some of the PIPs were linked to these drugs. Third, we limited alerts to DDIs classified by Micromedex ${ }^{\mathrm{TM}}$ Drug-Reax as producing severe adverse events with the aim of selecting interactions with a relatively high clinical relevance.

Despite these limitations, physicians involved in the study indicated a positive response, and the overall number of PIPs in both cohorts of frail older adults decreased significantly, although this might be subject to bias because participants were aware of the intervention and therefore might change their behavior in significant ways to alter the outcome.

\section{Conclusion}

Our study demonstrated that our system reduces the number of PIPs, improving medication appropriateness in hospitalized geriatric patients. Further prospective studies are warranted to evaluate the system's effectiveness in clinical practice.

\section{Compliance With Ethical Standards}

Funding No sources of funding were used to assist in the preparation of this article.

Conflict of interest Anna Maria Grion, Umberto Gallo, Daniel Dumitru Tinjala, Michele Loreggian, Giovanna Cardaci, Julia Daragjati, Arduino Mangoni, and Alberto Pilotto declare that they have no conflicts of interest relevant to the content of this article.

\section{References}

1. Beers MH, Ouslander JG, Rollingher J, et al. Explicit criteria for determining inappropriate medication use in nursing home residents. Arch Intern Med. 1991;151:1825-32.

2. Onder G, Landi F, Liperoti R, et al. Impact of inappropriate drug use among hospitalized older adults. Eur J Clin Pharmacol. 2005;61(5-6):453-9.

3. Gallagher PF, O'Mahony D. STOPP (Screening Tools of older Persons' Prescriptions): application to acutely ill elderly patients and comparison with Beers' criteria. Age Ageing. 2008;37:673-9.

4. Abarca J, Malone DC, Amstrong EP, et al. Concordance of severity ratings provided in four drug interaction compendia. J Am Pharm Assoc. 2004;44:136-41.

5. American Geriatrics Society. Beers Criteria Update Expert Panel. American Geriatrics Society updated Beers Criteria for potentially inappropriate medication use in older adults. J Am Geriatr Soc. 2012;60(4):616-31.

6. Gallagher P, Ryan C, Byrne S, et al. STOPP (Screening Tools of older Persons' Prescriptions) and START (Screening Tool to Alert Doctors to Right Treatment): consensus validation. Int $\mathrm{J}$ Clin Pharm Ther. 2008;45:72-83. 
7. Patterson SM, Cadogan CA, Kerse $\mathrm{N}$, et al. Interventions to improve the appropriate use of polypharmacy for older people. Cochrane Database Syst Rev. 2014. doi:10.1002/14651858. CD008165.pub3.

8. Tangiisuran B, Wright JE, van der Cammen TJM, et al. Adverse drug reactions in the elderly: challenges in identification and improving preventive strategies. Age Ageing. 2009;38:358-9.

9. Gillespie U, Alassaad A, Hammarlund-Udenaes M. Effects of pharmacists' intervention on appropriateness of prescribing and evaluation of the instruments' (MAI, STOPP and START) ability to predict hospitalization: analyses from a randomized controlled trial. PLOS One. 2013;8(5). doi:10.1371/journal.pone.0062401.

10. Ammenwerth E, Schnell-Inderst P, Machan C, et al. The effect of electronic prescribing on medication errors and adverse drug events: a systematic review. J Am Med Inform Assoc. 2008;15(5):585-600.

11. Weir CR, Staggers N, Laukert T. Reviewing the impact of computerized provider order entry on clinical outcomes: the quality of systematic reviews. Int J Med Inf. 2012;81(4):219-31.

12. Pilotto A, Ferrucci L, Franceschi M, et al. Development and validation of a Multidimensional Prognostic Index for one-year mortality from Comprehensive Geriatric Assessment in hospitalized older patients. Rejuvenation Res. 2008;11:151-61.

13. Siontis GC, Tzoulaki I, Ioannidis JP. Predicting death: an empirical evaluation of predictive tools for mortality. Arch Intern Med. 2011;171:1721-6.

14. Pilotto A, Panza F, Ferrucci L. A Multidimensional Prognostic Index in common conditions leading to death in older patients. Arch Intern Med. 2012;172:594-5.

15. Yourman LC, Lee SJ, Schonberg MA, et al. Prognostic indices for older adults. A systematic review. JAMA. 2012;307:182-92.

16. Taylor BH, Sketris I, Hayden J, et al. Application of the STOPP/ START criteria: a systematic review of the prevalence of potentially inappropriate prescribing in older adults and evidence of clinical, humanistic and economic impact. J Clin Pharm Ther. 2013;38:360-72.

17. Micromedex 2.0, Truven Health Analytics. Available from: http://www.micromedex.com.

18. Vitry AI. Comparative assessment of four drug interaction compendia. Br J Clin Pharmacol. 2006;63(6):709-14.

19. Medical Dictionary for Drug Regulatory Activities (MedDRA). Available from: https://www.meddra.org.

20. Hanlon JT, Schmader KE, Samsa GP, et al. A method for assessing drug therapy appropriateness. J Clin Epidemiol. 1992;45(10):1045-51.

21. R-Project for statistical computing (v.2.15.1, GNU Project). Available from: https://www.r-project.org.

22. Zhang J, Yu KF. What's the relative risk? A method of correcting the odds ratio in cohort studies of common outcomes. JAMA. 1998;280(19):1690-1.

23. Gallagher PF, O'Connor MN, O'Mahony D. Prevention of potentially inappropriate prescribing for elderly patients: a randomized controlled trial using STOPP/START criteria. Clin Pharmacol Ther. 2011;89(6):845-53.

24. Hamilton H, Gallagher P, Ryan C, et al. Potentially inappropriate medication defined by STOPP criteria and the risk of adverse drug events in older hospitalized patients. Arch Intern Med. 2011;171(11):1013-9.

25. Juurlink DN, Mamdani M, Kopp A, et al. Drug-drug interactions among elderly patients hospitalized for drug toxicity. JAMA. 2003;289:1652-8.

26. Tulner LR, Frankfort SV, Gijsen GJ. Drug-drug interactions in a geriatric outpatient cohort: prevalence and relevance. Drugs Aging. 2008;25(4):343-55.

27. Hines LE, Murphy JE. Potentially harmful drug-drug interactions in the elderly: a review. Am J Geriatr Pharmacother. 2011;9:364-77.

28. Vonbach P, Dubied A, Beer JH. Evaluation of frequently used drug interaction screening programs. Pharm World Sci. 2008;30:367-74.

29. Naugler CT, Brymer C, Stolee P, et al. Development and validation of an improving prescribing in the elderly tool. Can J Clin Pharmacol. 2000;7(2):103-7.

30. Lipton HL, Bird JA, Bero LA, et al. Assessing the appropriateness of physician prescribing for geriatric outpatients. Development and testing of an instrument. J Pharm Technol. 1993;9(3):107-13.

31. American College of Physicians. ACOVE quality indicators. Ann Intern Med. 2001;135:653-67.

32. Drenth van Maanen AC, van Marum RJ, Knol W, et al. Prescribing optimization method for improving prescribing in elderly patients receiving polypharmacy: results of application to case histories by general practitioners. Drugs Aging. 2009;26(8):687-701.

33. Basger BJ, Chen TF, Moles RJ. Inappropriate medication use and prescribing indicators in elderly Australians: development of a prescribing indicator. Drugs Aging. 2008;25(9):777-93.

34. Barrons R. Evaluation of personal digital assistance software for drug interactions. Am J Health Syst Pharm. 2004;61:380-5.

35. Knollmann BC, Dmith BJ, Garnett CE, et al. Personal digital assistant-based drug reference software as tools to improve rational prescribing: benchmark criteria and performance. Clin Pharmacol Ther. 2005;78(1):7-18.

36. Petrovic M, van der Cammen T, Onder G. Adverse drug reaction in older people. Detection and prevention. Drugs Aging. 2012;29(6):453-62.

37. Viktil KK, Salvesen HB, Reikvam A. The Janus face of polypharmacy: overuse versus underuse of medication. Norsk Epidemiol. 2008;18(2):147-52.

38. Budnitz DS, Lovegrove MC, Shebab N, et al. Emergency hospitalizations for adverse drug events in older Americans. N Engl J Med. 2011;365:2002-12.

39. Douglas IJ, Smeeth L. Exposure to antipsychotics and risk of stroke: self controller case series study. BMJ. 2008;337:a1227.

40. Coupland C, Dhiman P, Morriss R, et al. Antidepressant use and risk of adverse outcomes in older people: population based cohort study. BMJ. 2011;343:d4551.

41. Kaur S, Mitchell G, Vitetta L, et al. Interventions that can reduce inappropriate prescribing in the elderly: a systematic review. Drugs Aging. 2009;26(12):1013-28.

42. Topinková E, Baeyens JP, Michel JP, et al. Evidence-based strategies for the optimization of pharmacotherapy in older people. Drugs Aging. 2012;29(6):477-94. 